03,09

\title{
Тонкая структура ридберговских экситонов в закиси меди
}

\author{
(C) М.A. Семина
}

Физико-технический институт им. А.Ф. Иофрфе РАН, Санкт-Петербург, Россия

E-mail: msemina@gmail.com

\begin{abstract}
Экситоны большого радиуса, называемые также экситонами Ванье-Мотта, были обнаружены Е.Ф. Гроссом и Н.А. Каррыевым в 1952 г. Их энергетический спектр, отклик на внешние электрические и магнитные поля, а также на упругие деформации кристалла, активно изучались в 1960-70-е годы, Вторая волна интереса к экситонам в кристаллах $\mathrm{Cu}_{2} \mathrm{O}$ возникла сравнительно недавно в 2014 году, после наблюдения „гигантских“ высоковозбужденных состояний экситона в этом материале. Теоретическое описание высоковозбужденных состояний экситонов требует, как правило, новых подходов, поскольку для таких состояний становятся существенными отклонения от точно решаемой водородоподобной модели, а численное решение уравнения Шредингера с учетом особенностей зоной структуры кристалла становится исключительно ресурсоемкой задачей. В настоящей работе приводится краткий обзор недавних теоретических и экспериментальных исследований тонкой структуры энергетического спектра экситонов в закиси меди.
\end{abstract}

Работа была частично поддержана грантом РФФИ 15-52-12012 в рамках международной программы ДФГ-РФФИ ICRC TRR 160 и программами РАН.

DOI: $10.21883 /$ FTT.2018.08.46238.05Gr

\section{1. Введение}

Кулоновское взаимодействие между электроном и дыркой в полупроводниках приводит к образованию серии связанных состояний, экситонов, энергия которых лежит в запрещенной зоне. Плотность состояний электрон-дырочной пары перераспределяется между дискретным и сплошным спектром, поэтому эффекты притяжения носителей заряда важны и для их инфинитного движения. В результате, экситонные эффекты играют решающую роль в оптических свойствах полупроводников и полупроводниковых наносистем: квантовых ям, проволок и точек [1-4].

Зачастую экситоны могут быть описаны в рамках водородоподобной модели. Такое описание особенно распространено для полупроводников кубической симметрии, таких как $\mathrm{GaAs}$ или $\mathrm{Cu}_{2} \mathrm{O}$, спектр носителей заряда в которых в первом приближении является параболическим и изотропным. Однако отклонение энергетического спектра электронов и дырок от простой модели, характеризуемой скалярными эффективными массами, $\mathbf{k} \cdot \mathbf{p}$-смешивание различных зон, отклонение закона притяжения электрона и дырки от кулоновского за счет особенностей экранировки, обменное взаимодействие электрона и дырки приводят к количественным и качественным отклонениям экситонной серии от водородоподобной. Для основного и ближайших возбужденных состояний экситона вычислительные методы позволяют эффективно диагонализовать гамильтониан взаимодействующих электрона и дырки с учетом особенностей зонной структуры и специфики взаимодействия квазичастиц в полупроводниках, находить энергии связи и тонкую структуру спектра экситонов с достаточно высокой точностью [5-11].
Ситуация оказывается качественно иной для возбужденных состояний экситонов. Это обусловлено тем, что с увеличением главного квантового числа $n$, характеризующего наряду с орбитальным моментом $l$ и магнитным квантовым числом $m$ собственные функции водородоподобных состояний, резко $\propto n^{2}$ возрастает кратность вырождения мультиплета. Таким образом, прямой расчет экситонных волновых функций оказывается очень ресурсоемким. С другой стороны, именно возбужденные состояния экситонов с главными квантовыми числами $n \gtrsim 5$ привлекают особый интерес исследователей в последнее время. Изучение таких состояний позволяет провести аналогии с физикой ридберговских атомов, свойства которых в значительной мере отличаются от свойств состояний с малыми $n$ [12]. Необходимость исследования высоковозбужденных состояний экситонов ставит вопрос о разработке и применении новых методов для анализа свойств ридберговских экситонов.

В данной работе представлен краткий обзор новых результатов, полученных при исследовании современными как экспериментальными, так и теоретическими методами высоковозбужденных состояний экситонов ,желтой серии в объемных кристаллах закиси меди.

Закись меди является уникальным материалом, в котором сочетаются большая энергия связи экситона $\approx 87 \mathrm{meV}$ и высокое качество природных кристаллов. Именно на кристаллах $\mathrm{Cu}_{2} \mathrm{O}$ Е.Ф. Гросс и Н.А. Каррыев сделали открытие - впервые обнаружили проявления экситонов Ванье-Мотта в оптических спектрах [13]. Кристаллическая и зонная структура $\mathrm{Cu}_{2} \mathrm{O}$ показаны схематично на рис. $1, a$ и $b$, соответственно. Закись меди - центросимметричный кристалл с группой симметрии $\mathrm{O}_{\mathrm{h}}$. $\mathrm{B} \mathrm{Cu}_{2} \mathrm{O}$ зона проводимости и валентная зона 


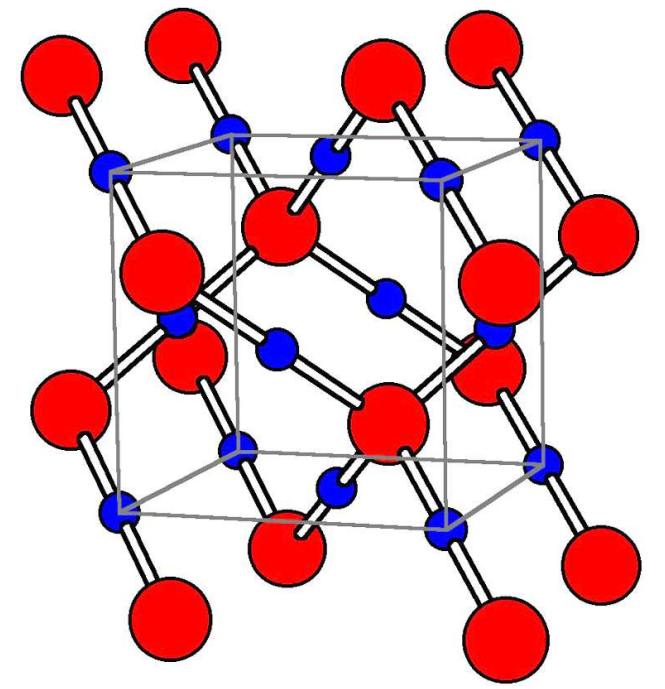

$a$

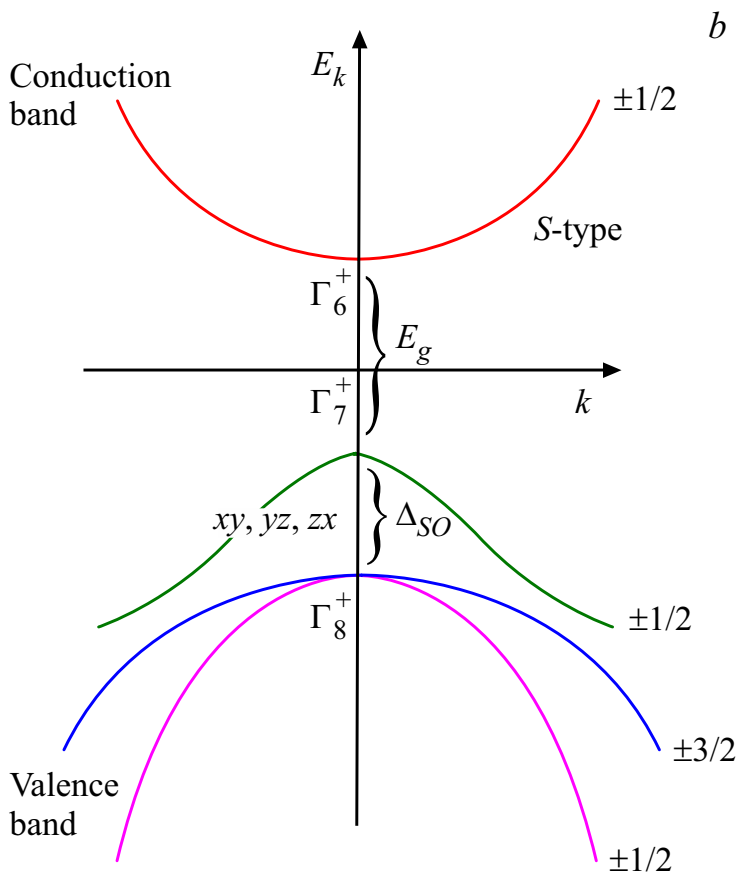

Рис. 1. (a) Кристаллическая и $(b)$ зонная структура закиси меди. Большие сферы - кислород, маленькие сферы медь. Зоны на панели $(b)$ маркированы соответствующими неприводимыми представлениями.

образованы орбиталями одинаковой четности $S$-типа $D$-типа, соответственно, что приводит к тому, что оптически активными в дипольном приближении являются $P$-состояния экситона, а не $S$-состояния, как в одном из самых популярных полупроводников GaAs. Таким образом, в экспериментах, использующих однофотонное возбуждение, в $\mathrm{Cu}_{2} \mathrm{O}$ наблюдаемая экситонная серия начинается с $n=2[13,14]$. Здесь и далее состояния экситона мы будет характеризовать набором квантовых чисел $n, l$ и $m$, соответствующих модели атома водорода. Строго говоря, квантовые числа $n, l, m$ не являются хорошими квантовыми числами для описания состояний в кубическом кристалле, поскольку состояния с $l \gtrsim 2$ преобразуются по приводимым представлениям группы $\mathrm{O}_{\mathrm{h}}$. Тем не менее, зачастую удобно использовать квантовые числа $n, l, m$ для обозначения базисных функций. Кроме того, в закиси меди расщепление между уровнями с разными $n$ превышает их тонкую структуру $[7,10,15]$.

Нас интересует „желтая“ экситонная серия, связанная с переходами между зоной проводимости, преобразующейся согласно представлению $\Gamma_{6}^{+}$группы $\mathrm{O}_{\mathrm{h}}$, и верхней валентной зоной, преобразующейся по представлению $\Gamma_{7}^{+}$. Несколько выше по энергии лежит „зеленая“ экситонная серия, связанная с переходами между зоной проводимости и спин-отщепленной валентной зоной, преобразующейся по представлению $\Gamma_{8}^{+}$. Необходимо отметить, что величина энергии связи основного состояния экситона сравнима с величиной спинового расщепления валентной зоны $\Delta_{S O}=134 \mathrm{meV}$ [16]. Это приводит к необходимости учета как валентной зоны $\Gamma_{6}^{+}$, так и валентной зоны $\Gamma_{8}^{+}$для описания тонкой структуры экситонных состояний [16].

\section{2. Тонкая структура экситонных состояний в отсутствие внешних полей}

Развитие современных методов спектроскопии позволило наблюдать состояния „желтой“ экситонной серии вплоть до $n=25$. Пример спектра поглощения показан на рис. 2, $a$ [17]. При его более подробном рассмотрении $[$ см. рис. $2, b]$ можно видеть с высокоэнергетичной стороны вблизи основных пиков, соответствующих $P$-состояниям начиная с $n=4$, дополнительные особенности, представляющие собой триплеты. Начиная с $n=6$ появляется еще одна особенность, тонкую структуру которой разрешить не удается [7].

Волновая функция экситона „желтой“ серии преобразуется по представлению $\mathscr{D}_{x}=\Gamma_{6}^{+} \times \Gamma_{7}^{+} \times \mathscr{D}_{r}$, где $\mathscr{D}_{r}$ - представление группы симметрии, отвечающее огибающей волновой функции относительного движения электрона и дырки. Здесь и далее мы рассматриваем экситоны в состоянии покоя, считая, что волновой вектор трансляционного движения электрон-дырочной пары, как целого, равен нулю. Дисперсии экситонов в объемных полупроводниках, а также поляритонным эффектам, неизбежным благодаря оптической активности экситонов, посвящен ряд работ [18-24]. Для того чтобы состояние было оптически активно в однофотонных процессах, в дипольном приближении его представление $\mathscr{D}_{x}$ должно содержать представление $\Gamma_{4}^{-}$, отвечающее компонентам вектора - оператора дипольного момента. Симметрийный анализ показывает, что в закиси меди в дополнение к $P$-состояниям экситона оптически активными будут $F$-, $H$ - и т.д. состояния с нечетной огибающей [7]. Для теоретического описания тонкой структуры спектра поглощения в закиси меди был проведен расчет экситонных состояний, в котором 


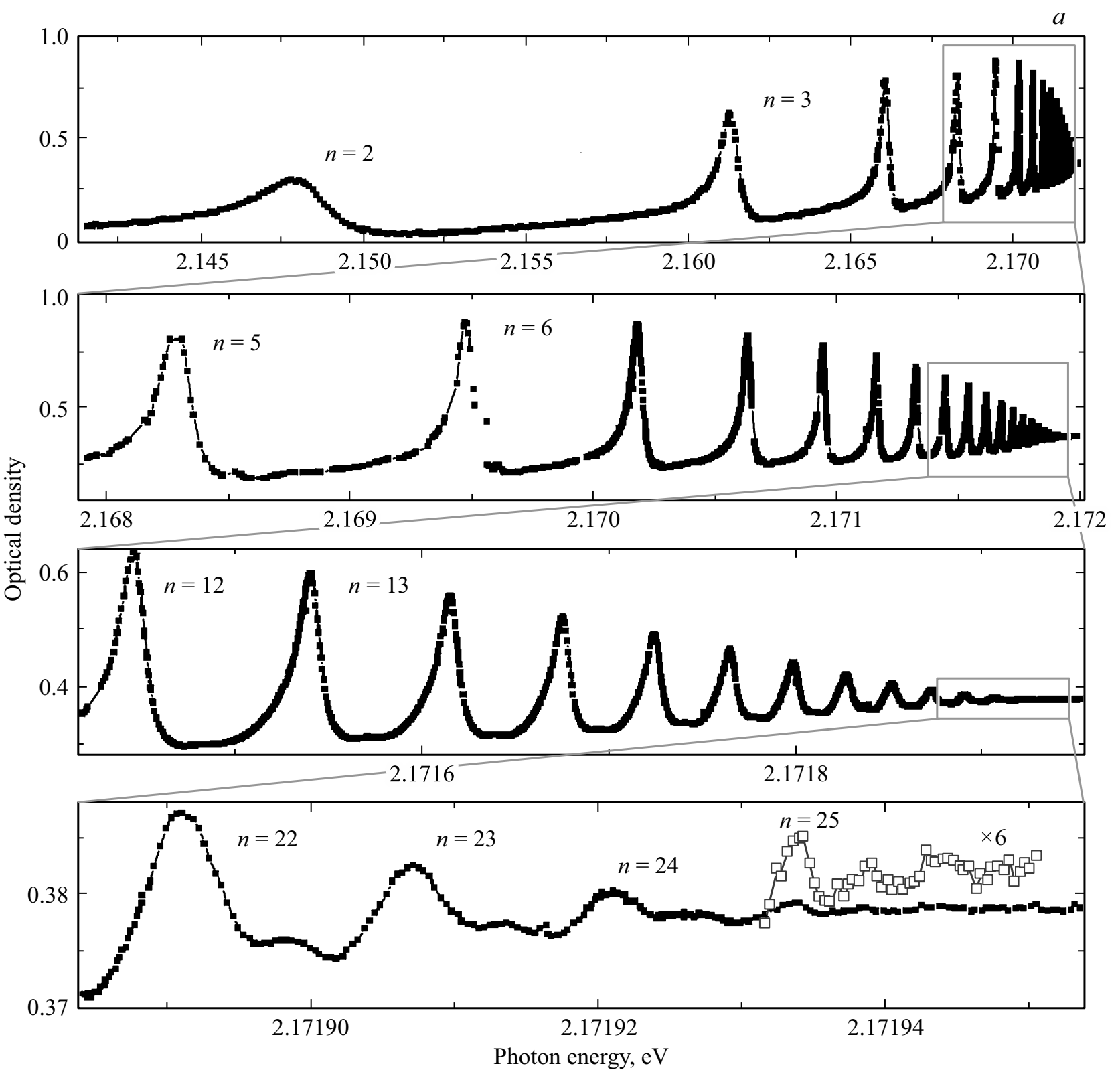

Рис. 2. (a) Спектр поглощения закиси меди [17]; (b) Спектр поглощения „желтой“ экситонной серии Сu $\mathrm{Cu}_{2} \mathrm{O}$ в диапазоне энергии, соответствующем состояниям с $n=4-9$. Верхние панели показывают высокоэнергетичную окрестность пиков, соответствующих $P$-состояниям экситонов с $n=4-7$ [7]; $(c)$ Зависимость энергии связи $P$-экситонов, а также $(d)$ расщепления между $P$ и $F$-состояниями экситона от номера уровня $n$. Точки показывают экспериментальные данные, результаты расчета показаны линиями, соединяющими точки, вычисленные для дискретных значений $n$. Параметры, использованные в расчете: $\mathscr{R}=87 \mathrm{meV}$, $\mu=\left(6 \gamma_{3}+4 \gamma_{2}\right) / 5 /\left(\gamma_{1}=m_{0} / m_{e}\right)=0.47, \Delta_{S O}=134 \mathrm{meV}[16]$.

были учтены как спин-отщепленная валентная зона $\Gamma_{8}^{+}$, так и кубическая симметрия кристалла. Для этого с использованием аппарата неприводимых тензоров был получен эффективный гамильтониан, описывающий относительное движение электрона и дырки

$$
\mathscr{H}=\frac{p^{2}}{\hbar^{2}}-\frac{2}{r}-\frac{\mu}{3 \hbar^{2}}\left(P^{(2)} \cdot I^{(2)}\right)+\frac{2}{3} \bar{\Delta}\left(1+\mathbf{I} \cdot \mathbf{s}_{h}\right)+\mathscr{H}_{c} .
$$

Здесь $\mathbf{p}$ - импульс относительного движения электрондырочной пары, I - матрицы оператора углового мо- мента $I=1$, действующего в базисе трех орбитальных блоховских амплитуд $y z, x z, x y$, преобразующихся по представлению $\Gamma_{5}^{+}, \mathbf{s}_{h}$ - оператор спина дырки. В (1) энергии измеряются в единицах $\mathscr{R}=e^{4} m_{0} /\left(2 \hbar^{2} \varepsilon^{2} \gamma_{1}^{\prime}\right)$, расстояния в единицах $a=\hbar^{2} \varepsilon \gamma_{1}^{\prime} /\left(e^{2} m_{0}\right), \varepsilon-$ статическая диэлектрическая проницаемость объемного кристалла, $\bar{\Delta}=\Delta_{S O} / \mathscr{R}$ - безразмерное спин-орбитальное расщепление зоны $\Gamma_{7}^{+}$и $\Gamma_{8}^{+}, \gamma_{1}^{\prime}=\gamma_{1}+m_{0} / m_{e}$, $\mu=\left(6 \gamma_{3}+4 \gamma_{2}\right) /\left(5 \gamma_{1}^{\prime}\right), m_{e}-$ масса свободного электрона, $\gamma_{i}(i=1,2,3)$ - параметры Латтинжера. По- 

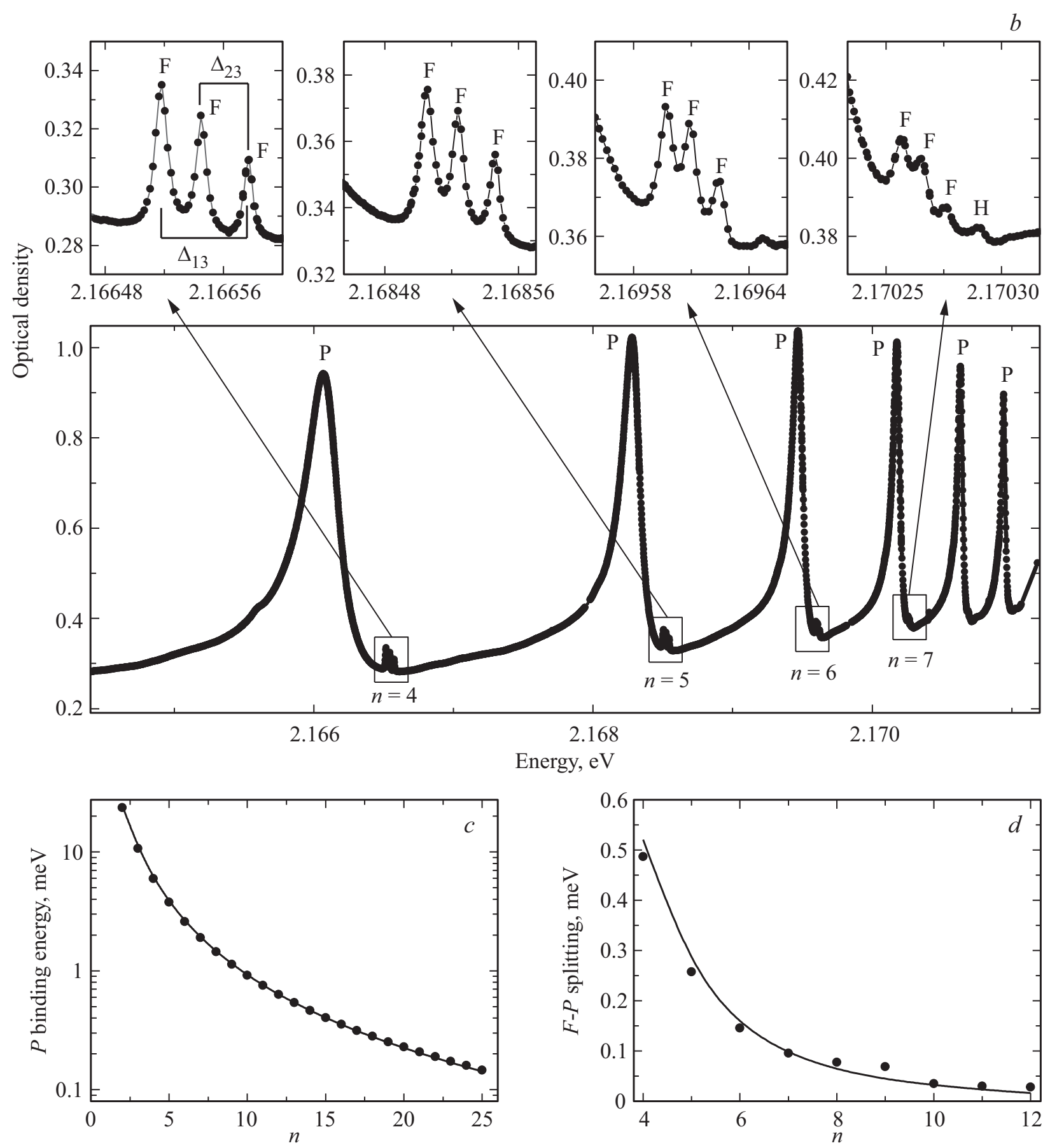

Рис. 2 (продолжение).

следний член в (1) учитывает кубическую симметрию кристалла

$$
\begin{gathered}
\mathscr{H}_{c}=\frac{\delta}{3 \hbar^{2}}\left(\sum_{k= \pm 4}\left[P^{(2)} \times I^{(2)}\right]_{k}^{(4)}+\frac{\sqrt{70}}{5}\left[P^{(2)} \times I^{(2)}\right]_{0}^{(4)}\right), \\
\delta=\left(\gamma_{3}-\gamma_{2}\right) / \gamma_{1}^{\prime} .
\end{gathered}
$$

В формулах (1) и (2) введены неприводимые тензоры второго ранга $P^{(2)}$ и $I^{(2)}$, составленные из произведений компонент оператора импульса $p_{\alpha} p_{\beta}$ и углового момента $I_{\alpha} I_{\beta}$, соответственно, $\alpha, \beta=x, y, z$.

На рис. 2, $c$ и $d$ показаны зависимости энергии связи $P$-состояний, а также расщепления между $P$-состоянием и центром триплета, связанного с $F$-состояниями. Для расчета использовались параметры из работы [16] без дополнительных подгоночных параметров. Как видно из рисунка, расчет дает хорошие согласие с экспериментом. Для описания тонкой структуры $F$-состояний был проведен расчет с одним подгоночным параметром $\delta$, харак- 
теризующим гофрировку валентной зоны. Сравнение с экспериментом позволило оценить значение параметра, определяющего гофрировку валентной зоны $\delta \approx-0.1$. Таким образом, получается, что $\delta$ имеет знак, противоположный знаку в распространенных материалах $\mathrm{Si}$, $\mathrm{Ge}, \mathrm{GaAs}$, однако это согласуется с расчетами микроскопическми методами [25] и с оценками параметров Латтинжера, выполненными в 1980-е годы на основе спектроскопии диамагнитных эффектов на экситонах и циклотронного резонанса в $\mathrm{Cu}_{2} \mathrm{O}[26,27]$. В первом расчете тонкой структуры $F$-экситонов в закиси меди [7] была допущена неточность, в результате чего состояния $F$-экситонов с большой и малой силой осциллятора были перепутаны. В работе [10] приведен уточненный расчет тонкой структуры возбужденных состояний экситона и показано, что наблюдаемые в эксперименте положения связанных с $F$-стояниями линий можно описать несколькими комбинациями параметров $\mu$ и $\delta$. На наш взгляд, требуются дальнейшие теоретические и экспериментальные исследования, в том числе детальные измерения сил осцилляторов $F$-экситонов, чтобы окончательно решить вопрос о параметризации валентной зоны в $\mathrm{Cu}_{2} \mathrm{O}$. Отметим, что расщепление состояний экситона с разным угловым моментом, например $P$ - и $F$ - состояний, является проявлением сложной структуры валентной зоны, в то время как тонкая структура $F$-состояний является следствием кубической симметрии материала.

\section{3. Скейлинг параметров экситонных состояний}

Кратность вырождения уровней экситона в рамках водородоподобной модели возрастает как квадрат номера уровня $n[28]$, поэтому детальное описание тонкой структуры высоковозбужденных состояний экситона представляет большую и часто не оправданную вычислительную сложность. Другим подходом является выявление общих закономерностей, в частности законов, согласно которым масштабируются свойства экситонных состояний с изменением номера уровня, его главного квантового числа. На наличие скейлинговых закономерностей указывает аналогия с атомной физикой, где показано, что экранировка кулоновского потенциала может быть включена в описание ридберговских атомов в виде простой модели квантовых дефектов, где энергия состояний с квантовыми числами $n$ и $l$ (без внешних полей сферическая симметрия атома приводит к вырождению состояний по магнитному квантовому числу) [12]

$$
E_{n l}=\frac{\mathscr{R}}{\left(n-\delta_{n, l}\right)^{2}} \approx \frac{\mathscr{R}}{n^{2}}+\frac{2 \delta_{l} \mathscr{R}}{n^{3}} .
$$

Здесь $\delta_{n, l}$ - квантовый дефект, который, как правило, мал по сравнению с единицей и слабо зависит от $n$. Таким образом, в частности, поправки к энергиям состояний масштабируются как $1 / n^{3}$. В работе [15] было отмечено, что с определенными ограничениями модель (3) применима и к экситонным состояниям в закиси меди, хотя природа квантовых дефектов существенно отличается от атомной. К отклонениям от водородоподобной серии состояний экситона в $\mathrm{Cu}_{2} \mathrm{O}$ приводит в основном сложная структура валентной зоны закиси меди, обусловленная спин-орбитальной связью, а также обменное взаимодействие между электроном и дыркой.

Анализ квантовых дефектов и скейлинговых закономерностей для экситонных состояний в закиси меди был выполнен в [29]. Для построения теоретической модели скейлинга параметров экситонных состояний использовалась теория возмущений. В качестве невозмущенного взят гамильтониан водородоподобной системы

$$
\widehat{H}_{0}=\frac{p^{2}}{2 \mu}-\frac{e^{2}}{\varepsilon r},
$$

где $\mu$ - приведенная масса экситона, $\varepsilon-$ диэлектрическая проницаемость. Энергия связи экситонных состояний с главным квантовым числом $n \geq 4$ оказвается $\lesssim 5 \mathrm{meV}$, что много меньше спинового расщепления валентной зоны $\Delta_{S O}=134 \mathrm{meV}$. Это делает возможным для состояний с $n \geq 4$ учитывать сложную структуру валентной зоны по теории возмущений, где поправки описываются следующим эффективным гамильтонианом:

$$
\widehat{H}_{d}=\mathscr{A} p^{4}+\frac{e^{2}}{\varepsilon r^{3}}\left[\mathscr{B}_{e}\left(\mathbf{l} \cdot \mathbf{s}_{e}\right)+\mathscr{B}_{h}\left(\mathbf{l} \cdot \mathbf{s}_{h}\right)\right]+C \delta(\mathbf{r})\left(\mathbf{s}_{e} \cdot \mathbf{s}_{h}\right) .
$$

Здесь первое слагаемое описывает непараболичность валентной зоны, второе - спин-орбитальное взаимодействие, третье - короткодействующее обменное взаимодействие между электроном и дыркой, $\mathscr{A}, \mathscr{B}_{e}, \mathscr{B}_{h}, C$ константы. В этом разделе мы отвлекаемся от кубической симметрии кристалла и используем сферическое приближение. Анализ в [29] показал, что все вклады в $\widehat{H}_{d}$ масштабируются с главным квантовым числом как $n^{-3}$, что делает возможным ввести по аналогии с атомной физикой для экситонов понятие квантового дефекта. Тогда энергия связи состояния с главным квантовым числом $n$ и орбитальным квантовым числом $l$ описывается формулой (3). Анализ показывает, что основной вклад в величину квантового дефекта дает непараболичность валентной зоны. Теоретическая оценка для величины

$$
\delta_{l} \approx \frac{8 \gamma_{2}^{2}}{m_{0}^{2} \Delta_{S O} \mathscr{R}(l+1)}\left(\frac{\hbar}{a_{B}}\right)^{4} .
$$

находится в хорошем согласии с экспериментом [29]. Подчеркнем, что несмотря на схожесть математического описания квантовых дефектов для экситонов и ридберговсих атомов, происхождение их различно. Для атомов наличие квантового дефекта связано главным образом с экранировкой потенциала ядра внутренней электронной оболочкой, в то время как для экситонов эффективный квантовый дефект - следствие сложной структуры валентной зоны. 
Для экситонов изучается скейлинг с главным квантовым числом следующих величин [29]:

- ширина мультиплета с заданным главным квантовым числом в отсутствие внешних полей;

- в магнитном поле - величина поля перехода к магнитоэкситону;

- величина магнитного поля, при котором происходит первый резонанс между соседними мультиплетами;

- в электрическом поле - величина поля, при котором происходит первый резонанс между соседними мультиплетами;

— энергия антипересечения мультиплетов в электрическом поле;

- поляризуемость экситонных состояний;

- величина электрического поля ионизации.

В качестве примера на рис. 3, a показана зависимость от главного квантового числа $n$ ширины мультиплета, состоящего из состояний с заданным главным квантовым числом [в теории эта ширина определяется по порядку величины вторым слагаемым в (3)], а на рис. $3, b-$ величины магнитного поля, при котором происходит резонанс между соседними мультиплетами. На рисунках символы показывают экспериментальные данные с учетом погрешности, сплошная кривая подгонка со скейлинговой зависимостью, полученной из теоретической модели, заштрихованная область - подгонка экспериментальных данных степенной функцией со свобободной степенью с учетом погрешности.

Как видно из формулы (3), используемая модель предсказывает зависимость ширины мультиплета $\sim n^{-3}$, фит экспериментальных данных дает зависимость $n^{-2.75 \pm 0.02}$, при этом кривая $\sim n^{-3}$ лежит в пределах экспериментальной погрешности. Такая скейлинговая зависимость полностью повторяет соответствующую зависиость для ридберговских атомов, за исключением атома водорода, в котором состояния в нулевом поле вырождены по величине орбитального момента. Экспериментальная зависимость для величины магнитного поля, в котором происходит первый резонанс между соседними мультиплетами, дает зависимость $B_{r} \sim n^{-3.72 \pm 0.08}$. В рамках водородоподобной модели величину поля резонанса можно оценить следующим образом. При достаточно больших $n$ работатет оценка $\left\langle r^{2}\right\rangle_{n l m}=\frac{n^{2}}{2}\left[5 n^{2}+1-3 l(l+1)\right] \propto n^{4}$, тогда энергия в магнитном поле может быть оценена как

$$
E_{n l} \approx-\frac{1}{n^{2}}+m B+A_{l} n^{4} B^{2}
$$

где $A_{l}$ - константа, зависящая от орбитального квантового числа $l$. Так как резонанс происходит между состояниями с $m=n-1$ и $m=-n$, то квадратичным по магнитному полю диамагнитным вкладом можно пренебречь, и мы получаем скейлинговую зависимость $B_{r} \sim n^{-4}$, которая, как видно из рисунка, лежит в пределах экспериментальной погрешности. Для ридберговских атомов скейлинговая зависимость иная: $B_{r} \sim n^{-6}$, что связано с тем, что из-за иных правил отбора
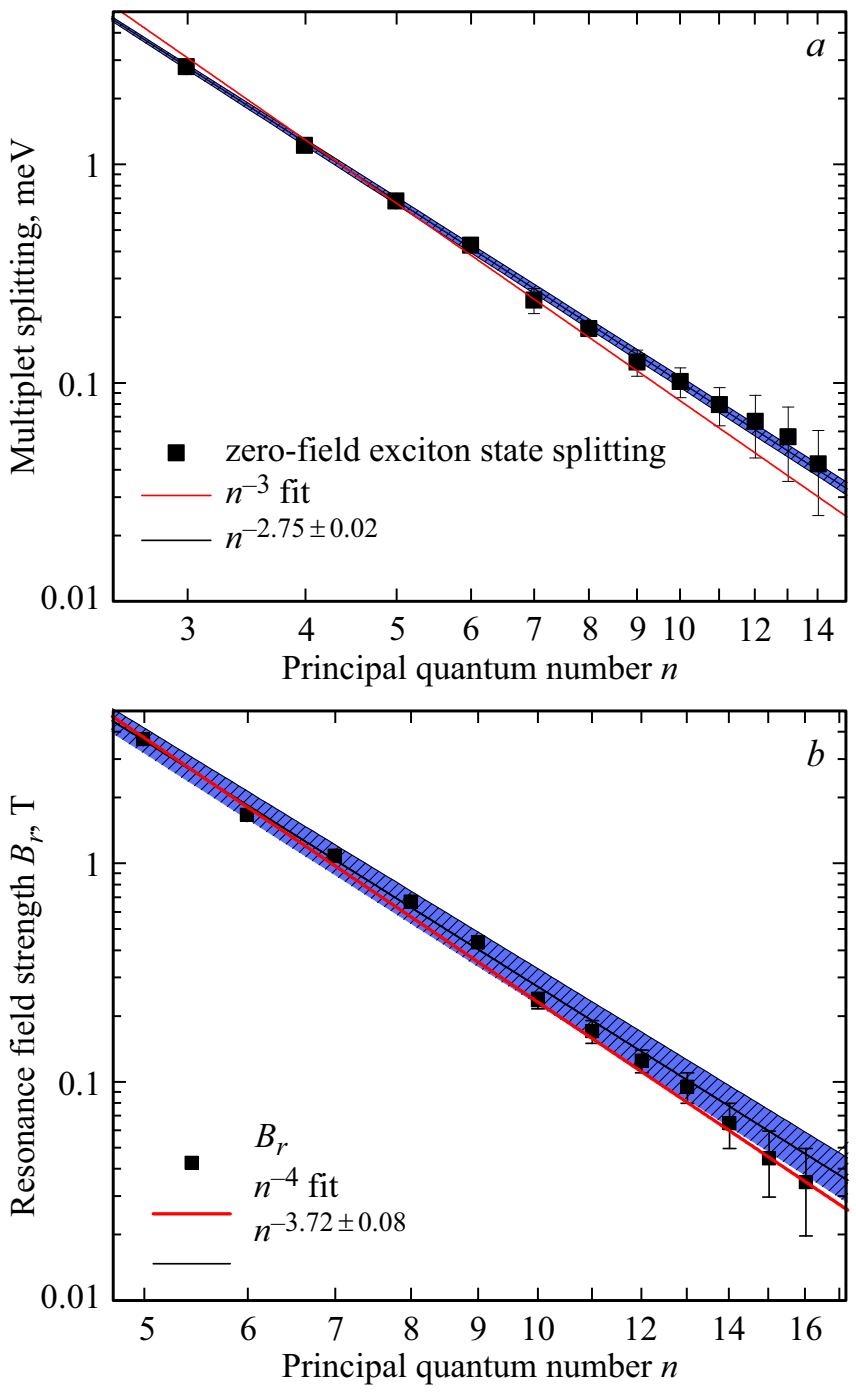

Рис. 3. Зависимость $(a)$ ширины мультиплета, состоящего из состояний с заданным главным квантовым числом, и $(b)$ величины магнитного поля, при котором происходит резонанс между соседними мультиплетами, от главного квантового числа $n$. Символы показывают экспериментальные данные с учетом погрешности, сплошная кривая - подгонка со скейлинговой зависимостью, полученной из теоретической модели, заштрихованная область - подгонка экспериментальных данных степенной функцией со свободной степенью с учетом погрешности.

при однофотонных переходах из $s$ состояния можно получить состояния только с малым $m=0, \pm 1$. При этом, напротив, главным является диамагнитный вклад в (7). Также был прослежен переход от кулоновского экситона к магнитоэкситону, когда электрон и дырка занимают свои уровни Ландау и квантуются независимо. Анализ экспериментальных данных, полученных в [29], показывает, что экспериментальные значения поля перехода $B_{c} \propto 1 / n^{3}$. Это соотношение легко получить из сопоставления расщепления между соседними мультиплетами $\sim \mathscr{R} / n^{3}$ и циклотронной энергии $\hbar|e| B / \mu c$. От- 
метим, что детальные расчеты (см., например, [30-32]) приводят к более жесткой оценке на поле $B_{c}^{\prime} \propto n^{-2}$. $\mathrm{B}$ таких полях $B \gtrsim B_{c}^{\prime}$ движение вдоль магнитного поля становится, по существу, одномерным. Второй переход при $B \gtrsim B_{c}^{\prime}$ для ридберговских экситонов в закиси меди пока не обнаружен в эксперименте. Однако, как продемонстрировано в экспериментах [33], для состояний с большими $n$ в магнитных полях реализуется квантовый хаос. В литературе обсуждаются разные механизмы возникновения хаоса [33-35].

\section{4. Экситоны в электрических полях}

Уже в ранних работах [14,36-42], последовавших за пионерской статьей Е.Ф. Гросса и Н.А. Каррыева, были выполнены исследования влияния внешних электрических и магнитных полей, а также упругих деформаций кристалла на спектр экситонов в закиси меди. Одним из ярких электрооптических явлений, обнаруженных на экситонах $\mathrm{Cu}_{2} \mathrm{O}$, было наблюдение оптической анизотропии кубического кристалла, связанной с проявлением квадрупольных переходов [43]. Ряд основных особенностей экситонных спектров и эффекта Штарка на экситонах в закиси меди был понят в 1970-е годы [37], однако развитие экспериментальных методик оптической спектроскопии с высоким разрешением и значительное продвижение в расчетах экситонных состояний открыли возможности количественного описания спектров экситонов в электрических полях.

Электрическое поле, как известно, смешивает состояния экситона с огибающими волновыми функциями относительного движения противоположной четности (с орбитальным квантовым числом, отличающимся на единицу), что делает оптически активными в дипольном приближении четные состояния, $S-, D-$ и т.д., неактивные в нулевом поле. Наличие сложной валентной зоны, спин-орбитального взаимодействия, обменного взаимодействия, кубической симметрии кристалла, и т.д., приводит к богатой тонкой структуре экситонных состояний в электрическом поле [8,9,37]. При этом мультиплеты, сформированные состояниями с разными главными квантовыми числами $n$, вообще говоря, нельзя рассматривать независимо: как отмечалось в разделе 3 для $n \geq 3$, расщепление между соседними состояниями оказывается достаточно малым, чтобы электрические поля, необходимые для пересечения состояний, происходящих из соседних мультиплетов, были легко достижимы в лабораторных условиях. Кроме того, в электрическом поле очень ярко проявляются отличия экситонных состояний от водородоподобной модели. Как хорошо известно [28], для кулоновского потенциала и параболических зон уравнение Шредингера относительного движения зарядов при наличии электрического поля допускает разделение переменных в параболической системе координат, это приводит к независимым сериям уровней от мультиплетов с различными $n$. Отклонения от водородоподобной модели приводят к антипересечению состояний (см. выше, а также $[8,29])$. Более того, в электрическом поле, вообще говоря, пропадает дискретный спектр состояний относительного движения, происходит ионизация экситонов. Параметрически поля, в которых проявляются антипересечения мультиплетов и ионизация экситонов, отличаются (их величины масштабируются с главным квантовым числом как $1 / n^{5}$ и $\left.1 / n^{4}\right)$, поэтому есть область полей, в которых эффектами ионизации можно пренебречь, а тонкая структура экситонных уровней уже очень богата.

В работе [8] были представлены результаты подобного экспериментального и теоретического исследования эффекта Штарка для экситона в закиси меди для состояний с $n=3-5$ в геометрии на пропускание. Для приложения электрического поля образец помещался в плоский кондесатор так, что электрическое поле было направлено вдоль оптической оси системы: $\mathbf{F} \| \mathbf{k}$, в противоположность ранним работам [14,36,37], в которых использовалась геометрия $\mathbf{F} \perp \mathbf{k}$. Результаты были получены для двух образцов, в обоих случаях внешнее электрическое поле было приложено вдоль нормали образца: $\mathbf{F}\|\mathbf{k}\|$ [001], для данной ориентации две ортогональные линейные поляризации света е $\|$ [100] и $\mathbf{e} \|[010]$ эквивалентны, и $\mathbf{F}\|\mathbf{k}\|[110]$, для которой поляризации света е $\|[001]$ и е $\|[0 \overline{1} 0]$ в кристалле с группой симметрии $O_{h}$ оказываются неэквивалентны.

Для теоретического описания экспериментальных результатов, полученных при изучении эффекта Штарка для экситона, была использована модель, сформулированная в разд. 3, которая учитывает расщепление состояний экситона с разными значениями орбитального квантового числа в нулевом поле. Спин-орбитальное и обменное взаимодействие, эффекты кубической симметрии кристалла, а также взаимодействие с электрическим полем учитывались по теории возмущений, описывающие их константы являлись подгоночными параметрами. Положения уровней энергии в нулевом поле были взяты из эксперимента. В расчете учтены все состояния экситона с $n=1-6$, в том числе смешивание между состояниями, происходящиим из мультиплетов с различными главными квантовыми числами. Анализ результатов показал существенную зависимость спектра экситона в электричеком поле как от ориентации электрического поля относительно кристаллических осей, так и от поляризации света в случае $\mathbf{F}\|\mathbf{k}\|$ [110].

В качестве примера на рис. 4 показаны экспериментальные данные и результаты расчета для состояния с $n=4$ для геометрии $\mathbf{F}\|\mathbf{k}\|[110]$ и $\mathbf{e} \|[001]$, при которых наблюдается максимальное число спектральных линий, так как в этом случае разрешены также квадрупольные переходы. При этом $S-$ и $D$-экситоны, которые смешиваются уже в нулевом электрическом поле, оказываются оптически активными [16]. Как видно из рисунка, результаты расчета хорошо описывают экспериментальные данные. При приложении электрического поля экситонные состояния расщепляются и сдвигаются. 

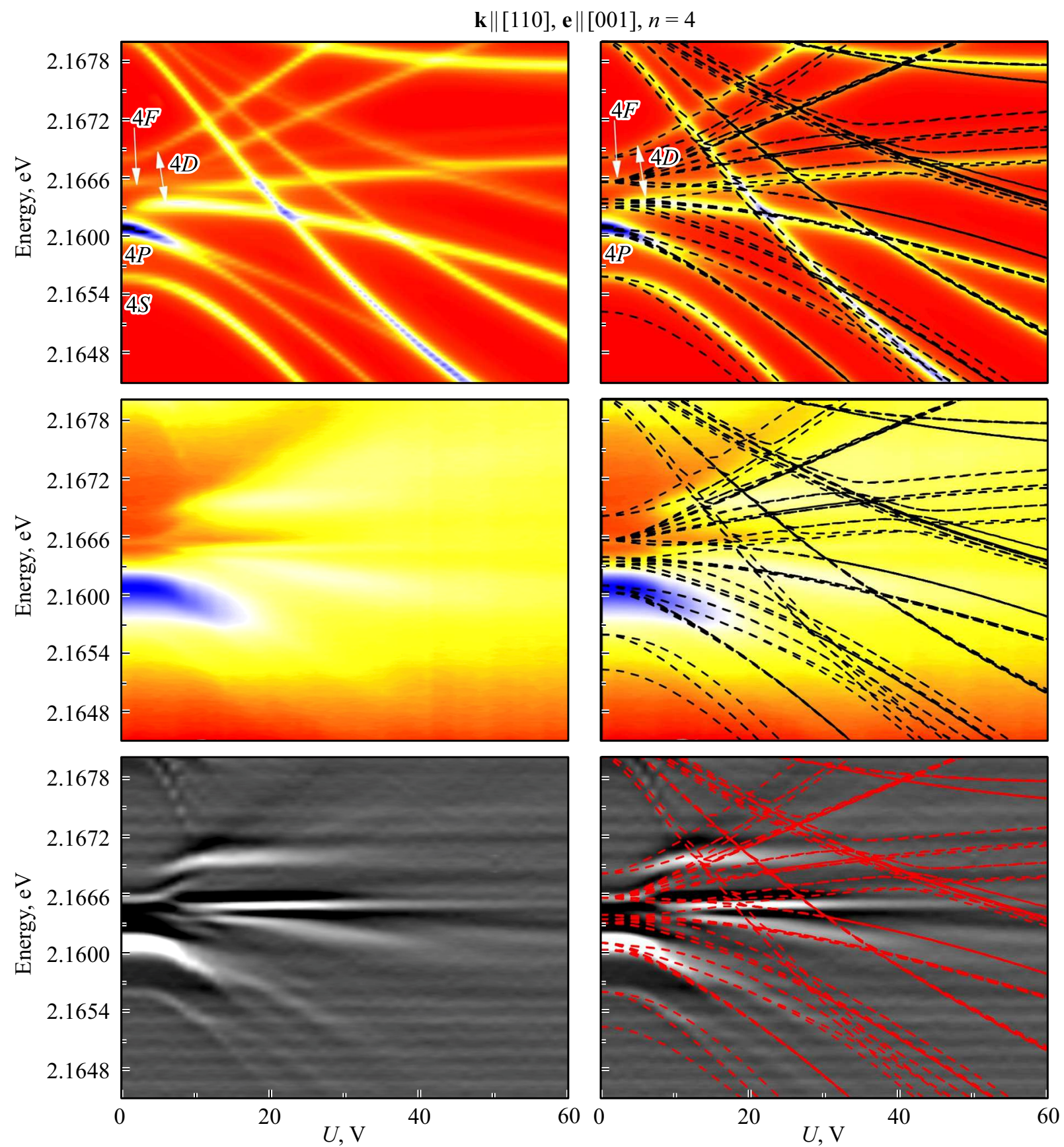

Рис. 4. Спектр поглощения в интервале энергий, соответствующих экситону с $n=4$ для геометрии $\mathbf{F}\|\mathbf{k}\|[110]$ и поляризации света е $\|$ [001]. Верхние панели - результаты расчета, средние - результаты эксперимента, нижние — вторая производная от измеренного спектра. На правых панелях штриховыми кривыми показаны собственные состояним экситона без учета правил отбора $[8]$.

Из рисунка также можно видеть, что при некотором приложенном напряжении происходит сближение верхних состояний с $n=4$ и нижних состояний с $n=5$.

Экспериментальные данные, приведенные на рис. 4, показывают, что помимо штарковских сдвигов и сложных антипересечений уровней, в достаточно сильных электрических полях линии экситонов резко уширяются и практически пропадают из спектра. Это есть свидетельство эффекта ионизации экситона: в сильном электрическом поле электрон и дырка могут туннелировать под барьером, сформированным кулоновским притяжением и внешним полем. Расчет спектра поглощения экситонов с учетом эффекта ионизации является исключительно сложной задачей, для этого, как правило, используется квазиклассическое приближение [28]. В полупроводниках с „разрешенным“ междузонным оптическим переходом задача об электропоглощении с учетом экситонного эффекта была решена Меркуло- 


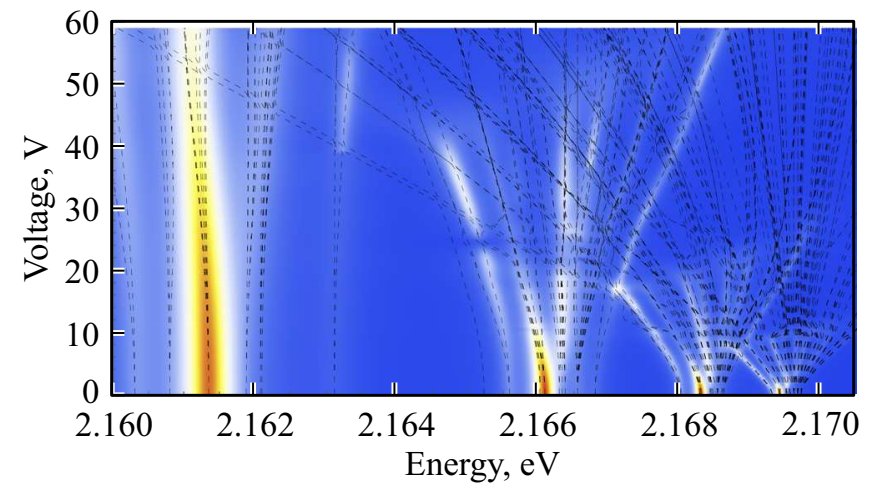

Рис. 5. Рассчитанный спектр пропускания закиси меди в геометрии $\mathbf{k}\|\mathbf{F}\|[$ [001], e $\|[100]$ для энергий вблизи состояний экситона с номерами $n=3-6$.

вым [44] и несколько более общим методом (позволяющим корректно найти коэффициент поглощения в области междузонных переходов в континуум) Ароновым и Иоселевичем [45]. Ионизация $P$-экситонов изучалась в последующей работе [46]. В этих статьях использовалась водородоподобная модель, а эффектами сложной структуры зон пренебрегалось. Отметим, что фактически параллельно с этим велись расчеты ионизации атома водорода, решение для состояний с произвольными $n$ и $l$ было получено С.Ю. Славяновым [47]. Учет отклонений спектра от водородоподобной серии исследовался, насколько нам известно, лишь для атомов в модели квантовых дефектов (см., например, [48]). Расчет спектров пропускания кристалла закиси меди в экситонной области спектра с учетом эффекта ионизации выполнен в [49] в модели квантовых дефектов. Спектр пропускания в геометрии $\mathbf{k}\|\mathbf{F}\|[001], \mathbf{e} \|[100]$ представлен на рис. 5. Видна сложная структура состояний и резкие модуляции интенсивности при пересечении ионизованных и еще не ионизованных состояний. Также расчет показывает, что внутри данного мультиплета состояние с большей энергией ионизуется в больших полях. Это связано с тем, что в таких состояниях эффективный дипольный момент направлен против электрического поля, а поэтому частицы оказываются дальше от туннельного барьера. Результаты расчетов подтверждаются экспериментальными данными $[29,49]$.

\section{5. Заключение}

Закись меди является классическим полупроводником, где экситонные эффекты изучаются уже более полувека. Развитие экспериментальных методик и вычислительных средств открыло возможности изучать высоковозбужденные состояния экситона, физические свойства которых существенно отличаются от хорошо исследованного основного и нескольких нижних уровней. Последние исследования экситонных эффектов в $\mathrm{Cu}_{2} \mathrm{O}$ позволили не только уточнить ряд параметров этого материала, но и привели к открытию новых явлений, включая квантовый хаос экситонных состояний в магнитных полях, скейлинговые закономерности экситонных параметров и неожиданную стабильность возбужденных состояний к внешнему полю.

Автор выражает признательность М. Ассману, М. Байеру, М.М. Глазову, Д. Фрелиху, Ю. Хекоттеру за ценные обсуждения.

\section{Список литературы}

[1] Excitons / Ed. E.I. Rashba, M.D. Sturge. North-Holland, Amsterdam (1982).

[2] C.F. Klingshirn. Semiconductor Optics. Springer, Berlin, (2012). $849 \mathrm{p}$.

[3] E.L. Ivchenko. Optical spectroscopy of semiconductor nanostructures. Alpha Science, Harrow UK (2005). 427 p.

[4] H. Haug, S.W. Koch. Quantum theory of the optical and electronic properties of semiconductors. 5th ed. World Scientic, Singapore (2009).

[5] A. Baldereschi, N. Lipari. Phys. Rev. B 8, 2697 (1973).

[6] A. Baldereschi, N.O. Lipari. Phys. Rev. B 9, 1525 (1974).

[7] J. Thewes, J. Heckötter, T. Kazimierczuk, M. Aßmann, D. Fröhlich, M. Bayer, M.A. Semina, M. M. Glazov. Phys. Rev. Lett. 115, 027402 (2015).

[8] J. Heckötter, M. Freitag, D. Fröhlich, M. Aßmann, M. Bayer, M.A. Semina, M.M. Glazov. Phys. Rev. B 95, 035210 (2017).

[9] S. Zielińska-Raczyńska, D. Ziemkiewicz, G. Czajkowski. Phys. Rev. B 94, 045205 (2016).

[10] F. Schweiner, J. Main, M. Feldmaier, G. Wunner, C. Uihlein. Phys. Rev. B 93, 195203 (2016).

[11] F. Schweiner, J. Main, G. Wunner, M. Freitag, J. Heckötter, C. Uihlein, M. Aßmann, D.Fröhlich, M. Bayer, Phys. Rev. B 95, 035202 (2017).

[12] T.F. Gallaher. Rydberg Atoms. Monographs on Nolecular and Chemical Physics. University Press, Cambridge (2005).

[13] Е.Ф. Гросс, Н.А. Каррыев. ДАН СССР 84, 471 (1952).

[14] E.F. Gross. Nuovo Cimento Suppl. 3, 672 (1956).

[15] F. Schöne, S.-O. Krüger, P. Grünwald, H. Stolz, S. Scheel, M. Aßmann, J. Heckötter, J. Thewes, D. Fröhlich, M. Bayer. Phys. Rev. B 93, 075203 (2016).

[16] C. Uihlein, D. Fröhlich, R. Kenklies. Phys. Rev. B 23, 2731 (1981).

[17] T. Kazimierczuk, D. Fröhlich, S. Scheel, H. Stolz, M. Bayer. Nature (London) 514, 343 (2014).

[18] M. Altarelli, N.O. Lipari. Phys. Rev. B 7, 3798 (1973).

[19] E.O. Kane. Phys. Rev. B 11, 3850 (1975).

[20] R. Benchamekh, F. Raouafi, J. Even, F.B.C. Larbi, P. Voisin, J.-M. Jancu. Phys. Rev. B 91, 045118 (2015).

[21] O. Roslyak, J.L. Birman. Solid State Commun. 145, 29 (2008).

[22] O. Roslyak, J.L. Birman. Phys. Rev. B 75, 245309 (2007).

[23] S. Zielińska-Raczyńska, G. Czajkowski, D. Ziemkiewicz. Phys. Rev. B 93, 075206 (2016).

[24] F. Schweiner, J. Ertl, J. Main, G. Wunner, C. Uihlein. Phys. Rev. B 96, 245202 (2017).

[25] E. Ruiz, S. Alvarez, P. Alemany, R.A. Evarestov. Phys. Rev. B 56, 7189 (1997); M. French, R. Schwartz, H. Stolz, R. Redmer. J. Phys.: Condens. Matter. 21, 015502 (2009).

[26] С.В. Гастев, Н.С. Соколов. ФТТ 22, 976 (1980); ФТТ 27, 416 (1985). 
[27] Н.Л. Яковлев, Н.С. Соколов. ФТТ 28, 1998 (1986).

[28] Л.Д. Ландау, Е.М. Лифшиц. Квантовая механика: нерелятивистская теория. Наука, М. (1974).

[29] J. Heckötter, M. Freitag, D. Fröhlich, M. Aßmann, M. Bayer, M.A. Semina, M.M. Glazov. Phys. Rev. B 96, 125142 (2017).

[30] А.Г. Жилич, Б.С. Монозон. ФТП 8, 3559 (1966).

[31] Р.П. Сейсян. Спектроскопия диамагнитных экситонов. Наука, М. (1984).

[32] Р.П. Сейсян. Диамагнитные экситоны в полупроводниках ФTT 58, 833 (2016).

[33] M. Aßmann, J. Thewes, D. Fröhlich, M. Bayer. Nature Mater. 15, 741 (2016).

[34] F. Schweiner, J. Main, G. Wunner. Phys. Rev. Lett. 118, 046401 (2017).

[35] F. Schweiner, J. Main, G. Wunner. Phys. Rev. E 95, 062205 (2017).

[36] V.T. Agekyan, B.S. Monozon, I.P. Shiryapov. Phys. Status Solidi B 66, 359 (1974).

[37] V.T. Agekyan. Phys. Status Solidi A 43, 11 (1977).

[38] Е.Ф. Гросс, Б.П. Захарченя, Н.М. Рейнов. ДАН СССР 97, 57 (1954); ДАН СССР 99, 527 (1954).

[39] Е.Ф. Гросс, Б.П. Захарченя, П.П. Павинский. ЖТФ 27, 2177 (1957).

[40] Е.Ф. Гросс. Успехи физических наук 63, 575 (1957).

[41] Е.Ф. Гросс, А.А. Каплянский. ФТТ 2, 2968 (1960).

[42] Е.Ф. Гросс. Успехи физических наук 76, 433 (1962).

[43] Е.Ф. Гросс, А.А. Каплянский. ФТТ 2, 379 (1960).

[44] И.А. Меркулов. ЖЭТФ 66, 6, 2314 (1974).

[45] А.Г. Аронов, А.С. Иоселевич. ЖЭТФ 74, 3, 1043 (1978).

[46] И.П. Арешев. ФТТ 21, 765 (1979).

[47] С.Ю. Славянов. Применение метода эталонных задач к возмущениям кулоновского поля. В сб.: Проблемы математической физики. Изд-воЛГУ, 4 (1970).

[48] В.Д. Мур, В.С. Попов. ЖЭТФ 94, 125 (1988).

[49] J. Heckötter, M. Freitag, D. Fröhlich, M. Aßmann, M. Bayer, M.A. Semina, M.M. Glazov. Dissociation of Rydberg excitons by an electric field to be published.

Редактор Ю.Э. Китаев 